\title{
Impaired endothelium-dependent and -independent relaxation of aorta from diabetic rats
}

\author{
Yakubu MA, Sofola OA, Igbo I, Oyekan AO \\ Vascular Biology Unit, Center for Cardiovascular Diseases, College of Pharmacy and Health Sciences, Texas \\ Southern University, Houston, USA. yakubu_ma@tsu.edu
}

\begin{abstract}
Vascular complication in diabetes has been reported to be due to the effects of chronic high blood glucose on the vascular system. Different relaxation mechanisms exist in the vasculature and effect of chronic high glucose on vascular relaxation mechanisms is not clearly understood. We assessed the effect of streptozotocin (STZ, $70 \mathrm{mg} / \mathrm{kg}$, for $12 \mathrm{wks}$ )-induced diabetes on vascular reactivity to isoproterenol (Isop, $10^{-9}-10^{-5} \mathrm{M}$ ), a cAMPdependent agent, acetylcholine (ACh, $10^{-9}-10^{-5} \mathrm{M}$ ), a stimulant of $\mathrm{NO}$ (nitric oxide) synthase, sodium nitroprusside (SNP, $10^{-10}-10^{-5} \mathrm{M}$ ), NO donor, or bradykinin $\left(\mathrm{BK}, 10^{-9}-10^{-5} \mathrm{M}\right)$ in the rat isolated aortic ring. Isop, ACh, SNP, or BK dose-dependently relaxed phenylephrine $\left(\mathrm{PE}, 10^{-7} \mathrm{M}\right)$ pre-constricted ring producing a maximum relaxation of 82 $\%$ for Isop $\left(10^{-5} \mathrm{M}\right), 85 \%$ for ACh $\left(10^{-5} \mathrm{M}\right), 100 \%$ for SNP $\left(10^{-6} \mathrm{M}\right)$, and $30 \%$ for BK $\left(10^{-5} \mathrm{M}\right)$ respectively. STZ attenuated Isop, ACh, and BK-induced relaxation by $45 \%(n=7, p<0.05), 38 \%(n=9, p<0.05)$, and $19 \%(n=7$, $p<0.05)$ respectively. Tension-induced by PE was significantly greater in STZ rats. SNP-induced relaxation was enhanced at the lower concentrations (from $10^{-10}-10^{-8} \mathrm{M}$ ) in the STZ treated group. These data suggest that conditions involving chronic high glucose sensitize the vasculature to vasoconstriction and selectively impairs vascular relaxation mechanisms that involve cGMP, NOS, but not that mediated by cAMP. Thus, STZ-induced impaired vasodilation may be mediated via chronic hyperglycemia-induced alteration of the vascular smooth muscle receptor proteins that may involve that recognized by BK, isoproterenol as well as their second messenger systems in addition to impairment of NO-dependent system. These findings suggest that distinct mechanisms may mediate the impaired responses to endothelium-independent agonists and such impairment could be due to the extent and duration of blood glucose dysregulation (Fig. 5, Ref. 24). Full Text in PDF www.elis.sk.

Key words: streptozotocin, endothelium, nitric oxide, ACh bradykinin, isoproterenol relaxation.
\end{abstract}

Different mechanisms mediate vascular relaxations to vasoactive agents. These mechanisms may be selectively compromised following pathological conditions in vascular diseases. In the periphery, diabetes-induced vascular dysfunction has been noted to be characterized by alterations in nitric oxide (NO) biosynthesis, release, or bioactivity (Taylor et al, 1995). Changes in the biosynthetic process of NO have formed the bedrock of the endothelial basis of vascular dysfunction following chronic hyperglycemia. Most studies that examined the impairment of endothelial vasodilator function associated with diabetes have focused on the role of NO. And the large majority of these studies demonstrate an impaired vasodilatation to endothelium-dependent agonists in the presence of preserved responses to endothelium-independent vasodilatators. This suggests that the diabetic state does not cause a generalized reduction in the sensitivity of the smooth muscle to endothelium-derived relaxing factors. Such assumptions often lead

Vascular Biology Unit, Center for Cardiovascular Diseases, College of Pharmacy and Health Sciences, Texas Southern University, Houston, USA

Address for correspondence: Momoh A. Yakubu, PhD, Vascular Biology Unit, Center for Cardiovascular Diseases College of Pharmacy and Health Sciences, Texas Southern University, 3100 Cleburne Avenue, Suite 101 Gray Hall, Houston, TX 77004, USA.

Phone: +713.3134231, Fax: +713.3134342

Acknowledgement: This study was supported by grants from the National Institute of Health: HL-70669, TSU Research Scientist Award (HL03674). to conflicting conclusions (De Vriese et al, 2000). In a few studies, decreased responses to nitrovasodilators have been reported in diabetic rats (Dai et al, 1993). However, information on the effects of chronic elevation of glucose on other dilatory mechanisms other than the endothelium-dependent mechanism is limited and not so certain. In the vascular system, dilatory processes mediated through prostanoids, cAMP, hyperpolarizing factors, or cGMP are known to play prominent roles in maintaining the vascular integrity. But the role of these dilatory pathways in the development of diabetic vasculopathy is still not clearly understood-especially the mechanisms that have their origin in the vascular smooth muscle cells. We have investigated the hypothesis that chronic hyperglycemiainduced by STZ impaired vascular dilatory mechanisms beyond the NO-/endothelium dependent dilatory signals.

\section{Materials and methods}

Experimental animals: Male Sprague-Dawley rats (300$450 \mathrm{mg}$ ) were used for this study. The protocol for this study was approved by the Animal Care Committee of the Texas Southern University, Houston. Rats were maintained according to the NIH Guidelines on Care for Animal Use in Research.

Induction of diabetes: Diabetes was induced in rats by a single dose injection of streptozotocin (STZ; $70 \mathrm{mg} / \mathrm{kg}$; ip), control rats received equivalent volume of vehicle. The rats were maintained 
for 12 weeks with access to water and food ad libitum before vascular reactivity to vasoactive agents was conducted.

Effects of diabetes on vascular reactivity: Following anesthesia with urethane (1 mg/kg; ip), the chest cavity was opened, and the thoracic aorta removed and placed in a petri-dish containing cold Krebs' $\left(4{ }^{\circ} \mathrm{C}\right)$ solution of the following composition $(\mathrm{mM})$ : $\mathrm{NaCl} 113, \mathrm{KCl} 4.7, \mathrm{NaHCO}_{3} 25.0, \mathrm{CaCl}_{2} 2.5, \mathrm{KH}_{2} \mathrm{PO}_{4} 1.2, \mathrm{MgSO}_{4}$ 1.2 , and glucose $5, \mathrm{pH} 7.4$, and continuously gassed with $95 \%$ $\mathrm{O}_{2}, 5 \% \mathrm{CO}_{2}$. The aorta was cleansed of connective tissue and cut into 3-4 mm rings. The aortic ring was then mounted in a $10 \mathrm{~mL}$ jacketed bath (World Precision Instruments-WPI, Sarasota, FL) at $37^{\circ} \mathrm{C}$. The ring was suspended in bath solution by means of two hooks, the lower one fixed to the bottom of the bath while the upper one was connected via a transbridge (model TBM4, World Precision Instruments, Sarasota, FL) data-acquisition system (DataQ Instruments, Akron, $\mathrm{OH}$ ) for recording of isometric tension developed to application of vasoactive agents. The rings were subjected to a resting tension of $2 \mathrm{~g}$ and allowed to equilibrate for a period of $90 \mathrm{~min}$ while being rinsed every $15 \mathrm{~min}$. During the equilibration period, the rings were subjected to two challenges of $10^{-7} \mathrm{M}$ phenylephrine (PE), 30 min apart.

Cumulative dose-response curves: Following equilibration, aortic rings were pre-constricted with $\operatorname{PE}\left(10^{-7} \mathrm{M}\right)$. The concentration of $\mathrm{PE}$ used was shown in preliminary experiments to produce about $70 \%$ of maximal contraction. The contraction to PE was allowed to reach a plateau and stabilize $(5-7 \mathrm{~min})$ before relaxation studies commenced. Relaxation responses to cumulative doses of acetylcholine (ACh), bradykinin (BK), sodium nitroprusside (SNP), or isoproterenol $\left(10^{-9}-10^{-5} \mathrm{M}\right)$ were determined. Changes in tension were monitored via a force displacement transducer connected to a DI-720 system (DATAQ software).

Statistical Analysis: Vascular relaxation responses are presented as $\%$ change in relaxation of aortic ring from pre-constricted values. Data are reported as mean \pm SEM and subjected to analysis of variance (ANOVA) followed by Student Newman-Keul's post-hoc test. $\mathrm{P}<0.05$ was considered significant.

\section{Results}

Aortic ring from STZ rats developed significantly greater spontaneous tension compared to the control and PE-induced tension was significantly greater in this group of rats. Following PE $10^{-7}$ $M$ tension generated by the aortic rings was $901 \pm 51$ grams in the control vs $1142 \pm 99$ grams in STZ rats (Fig. 1) $(\mathrm{p}<0.05 ; \mathrm{n}=21)$.

Figure 2 depicts the effects of STZ-induced diabetes on the vascular relaxations to BK $\left(10^{-10}-10^{-5} \mathrm{M}\right)$. In the control BK dosedependently relaxed $\mathrm{PE}\left(10^{-7} \mathrm{M}\right)$ pre-contracted aortic rings, the relaxations were significantly $(\mathrm{p}<0.05)$ attenuated in the rings from STZ rats by shifting the graph to the left. In the control group, the highest concentration of BK $\left(10^{-5} \mathrm{M}\right)$ employed relaxed the PEinduced tension by $41 \pm 6 \%$ compared to the $21 \pm 4 \%$ in the STZ group $(\mathrm{p}<0.05 ; \mathrm{n}=6-12)$.

Figure 3 shows the effects of STZ on the relaxations to isoproterenol $\left(10^{-10}-10^{-5} \mathrm{M}\right)$, dose-dependent relaxations to isoproterenol in the control were attenuated $(\mathrm{p}<0.05)$ by STZ treatment shifting

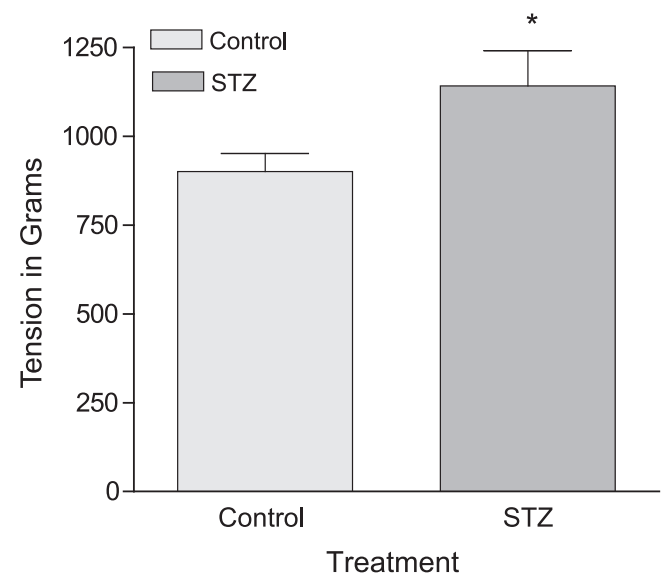

Fig. 1. Effects of STZ-induced diabetes on the vascular contraction to PE $\left(10^{-7} \mathrm{M}\right)$. Data are presented as mean \pm sem; $* \mathbf{p}<0.05$ compared to the control, $t-T e s t, \mathbf{n}=\mathbf{2 1}$ from different rats.

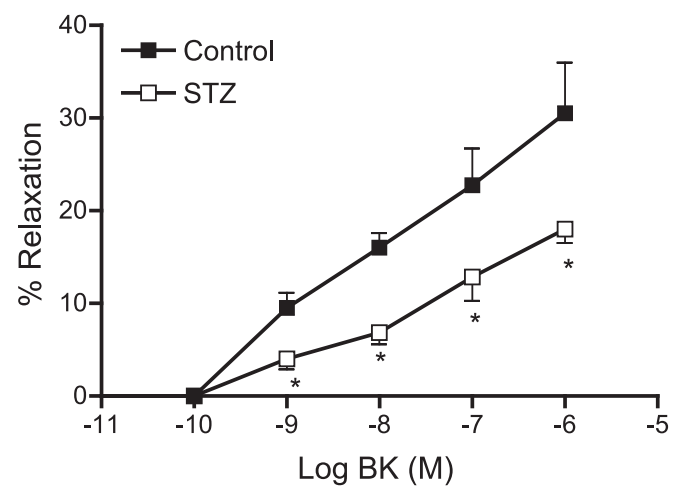

Fig. 2. Effects of STZ-induced diabetes on the vascular relaxations to BK $\left(10^{-9}-10^{-5} \mathrm{M}\right)$ of aortic ring pre-constricted with $\mathrm{PE}\left(10^{-7} \mathrm{M}\right)$. Data are presented as mean \pm sem; * $\mathbf{p}<0.05$ compared to the control, * $p<0.05$ compared to control, ANOVA, $n=7$ from different rats.

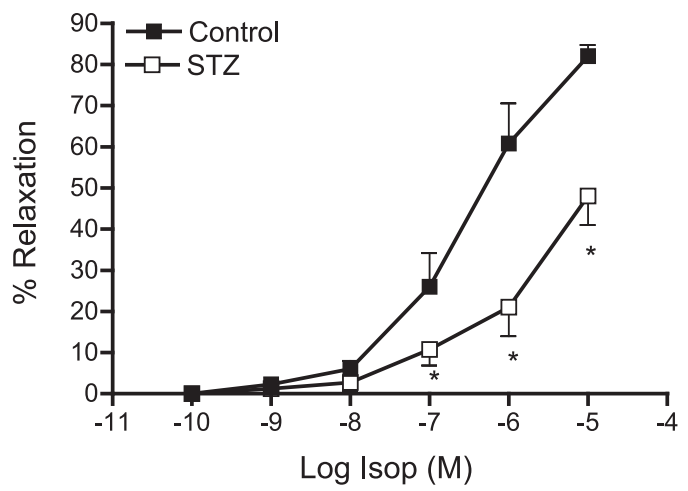

Fig. 3. Effects of $\mathrm{STZ}$ on the relaxations to isoproterenol $\left(10^{-9-10^{-5}} \mathrm{M}\right)$, of aortic ring pre-constricted with PE $\left(10^{-7} \mathrm{M}\right)$. Data are presented as mean \pm sem; * $\mathbf{p}<0.05$ compared to the control, * $\mathbf{p}<0.05$ compared to control, ANOVA, $n=7$ from different rats.

the graph to the left. The highest concentration of isoproterenol employed relaxed the PE pre-constricted rings by $82 \pm 3 \%$ compared to $48 \pm 7 \%$ relaxation observed in the $\mathrm{STZ}$ group $(\mathrm{p}<0.05 ; \mathrm{n}=6)$.

Figure 4 shows the effects of STZ on the relaxation of aortic rings induced by application of ACh. In the control ACh dose- 


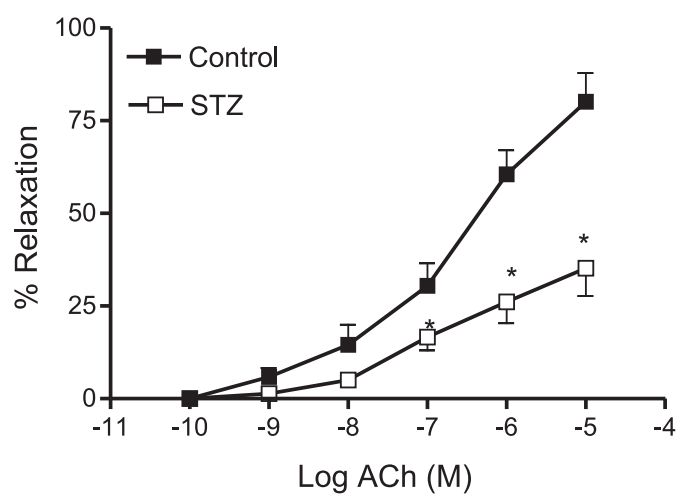

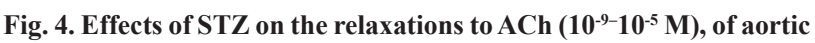
ring pre-constricted with $\mathrm{PE}\left(10^{-7} \mathrm{M}\right)$. Data are presented as mean \pm sem; * $p<0.05$ compared to the control, $* * \mathbf{p}<0.05$ compared to control, ANOVA, $n=7$ from different rats.

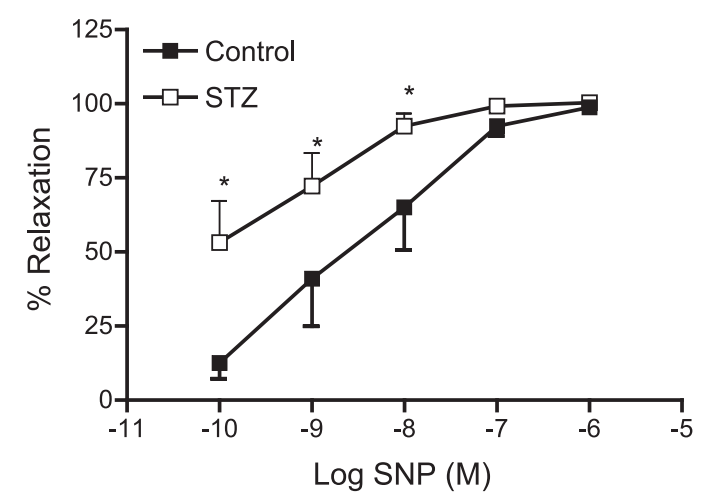

Fig. 5. Effects of $S T Z$ on the relaxations to $\operatorname{SNP}\left(10^{-10-10^{-6}} \mathrm{M}\right)$, of aortic ring pre-constricted with $\mathrm{PE}\left(10^{-7} \mathrm{M}\right)$. Data are presented as mean \pm sem; * $\mathbf{p}<0.05$ compared to the control, $* * \mathbf{p}<0.05$ compared to control, ANOVA, $n=7$ from different rats.

dependently relaxed PE-induced contraction of the aortic rings; these relaxations were significantly attenuated $(\mathrm{p}<0.05)$ in the rings from diabetic rats. The graph was shifted to the left with the highest concentration of ACh $\left(10^{-5} \mathrm{M}\right)$ employed causing $80 \pm 8 \%$ in the control as compared to $35 \pm 8 \%$ in the STZ treated group $(\mathrm{p}<0.05 ; \mathrm{n}=6)$.

Figure 5 shows the effects of STZ on relaxation of the aortic ring to SNP. In the control, SNP dose-dependently relaxed PE-induced tension, the relaxation was significantly enhanced $(\mathrm{p}<0.05)$ at the lower concentrations in the STZ group. The graph was shifted to the right by producing enhanced relaxations of $53 \pm 14 \%$ in STZ vs $13 \pm 5 \%$ in the control at $10^{-10} \mathrm{M}, 72 \pm 11 \%$ in STZ vs $41 \pm 16 \%$ in the control at $10^{-8} \mathrm{M}, 92 \pm 4 \%$ in STZ vs $62 \pm 14 \%$ in the control at $10^{-8} \mathrm{M}(\mathrm{p}<0.05 ; \mathrm{n}=5)$. The rightward shift of the graph in STZ rat was normalized at the higher concentrations of SNP employed.

\section{Discussion}

The key findings of the present study are that, in addition to the well reported diabetes-induce endothelial dysfunction characterized by attenuation of NO-mediated endothelium-dependent relaxation, we now report that STZ: 1) also attenuated isoproterenol and bradykinin but not forskolin-induced relaxations, 2) SNP-induced relaxations at the lower concentrations were enhanced shifting the dose-response curve rightward, and 3) ACh, Isoproterenol, and BK resistant tensions were relaxed by SNP or forskolin. Thus, STZ-induced impaired vasodilation may be mediated via chronic hyperglycemia-induced alteration of the vascular smooth muscle receptor proteins that may involve that recognized by BK, isoproterenol as well as their second messenger systems in addition to impairment of NO-dependent system. These findings suggest that distinct mechanisms may mediate the impaired responses to endothelium-independent agonists and such impairment could be due to the extent and duration of blood glucose dysregulation. Thus, our present results are consistent with that reported in humans and that such duration of treatment is necessary to provide comparable disease conditions in humans.

In the vascular system, multiple pathways mediate vascular dilation. These pathways involve both endothelial and nonendothelial derived vasoactive agents (Hoepfl, 2002; Cines et al, 1998; Ignarro, 1990; Moncada et al, 1991). The endothelium derived agents play an important role in the maintenance of vascular tone; the agents produced by the endothelial cell interact with the smooth muscle cells to cause relaxation (Moncada et al, 1991; Cines et al, 1998). The agent-smooth muscle cell interact generate secondary message culminating in the dilation of vascular bed. Principal among the dilatory agents produced and released by the endothelium includes nitric oxide, prostacyclin, endothelium-derived hyperpolarizing agents etc (Moncada et al, 1991; Cines et al, 1998; Hoepfl et al, 2002). The relative importance and vulnerability to modulation of these dilatory pathways under physiological and pathphysiological condition varies and depend on their individual characteristics. . NO has been shown to be one of the most important and widely regulated of these agents, especially in the large vessels ((Moncada et al, 1991; Cines et al, 1998; Chan et al, 2000; Ignarro 1990).

Under pathological conditions, selective alteration of different vascular dilatory mechanisms may result. The severity of such alteration depends on numerous factors, principal among which is the duration and severity of the perturbing factors, and the vulnerability of the dilatory mechanism that may be altered. Other vasoactive agents not produced by the endothelium may activate membrane receptors and/ or second messengers on the vascular smooth muscle to cause dilation. All these mediators may become target for regulation under different conditions. Acute and to some extent chronic high glucose has been reported to alter NO-dependent relaxation (Sobrevia and Mann, 1997; Taylor et al, 1995; Tesfamariam et al, 1991, Tesfamariam and Cohen, 1992, Gutterman 2002). But, relatively none or very few information is available on the effects of chronic high glucose on NO-/ endothelium-independent relaxations. We had previously reported that acute high glucose resulted in attenuated ACh-mediated dilation, while relaxations to SNP and isoproterenol were unaffected (Yakubu et al, 2003). In the present study, we found that following chronic high glucose in STZinduced diabetic rats, endothelium-dependent dilation mediated by NO as well as non-endothelium-dependent mediated relaxations to isoproterenol and bradykinin were significantly impaired but 
not those relaxations to SNP and forskolin. The results presented show that following prolong chronic exposure of the vasculature to high glucose; impairment of the vascular function spread beyond the endothelial dysfunction to none endothelial mediated dilatory mechanisms. The present finding is very significant as it shows that vascular complications observed in diabetes cannot be completely explain by the endothelial dysfunction hypothesis. The present results shows that following chronic exposure of the vasculature to hyperglycemic conditions, dysfunction of the vascular system goes beyond the endothelium and probably involves non-NO/nonendothelium dependent dilatory processes. In the present study, chronic hyperglycemia apart from attenuation of NO-mediated relaxation, simultaneously argument vascular smooth muscle responsiveness to SNP. This augmentation is presumably mediated via a cGMP-mediated mechanism. Enhanced cGMP reactivity can result following chronic suppression of NO production or availability in the presence of SNP a NO donor akin to starvation hypersensitivity of the vascular smooth muscle.

The mechanism behind STZ-induced diabetes alteration of vascular function has been the subject of investigation for a long time and results from such studies have mostly reported dysfunction of endothelium and /or dependent vasodilation (ref). Various mechanisms have been proposed for the vascular dysfunction observed and one of the principal mechanisms advanced has been increased oxidative stress. Increased oxidative stress has been the hallmark of diabetes and consequent generation of free radicals has been widely reported (Brodsky et al, 2001; Cosentino et al, 1997; Giuliano et al, 1996; 1997; Inoguchi et al, 2000). Interactions of NO with the excessively produced oxygen radicals neutralize the NO dilatory mechanism resulting in blunted vascular dilation. The attenuated effects could have occurred as a result of reduction in NO bioavailability and/ or reduction in NO synthetic process. The first scenario is the most plausible in acute exposure to glucose, and that is why most reports of vascular dysfunction in diabetes focuses on the dysregulation of NO-mediated vascular dilation (Rubyanyi and Vanhoutte, 1986; Sobrevia and Mann, 1997). The NO-system is easily compromised in the presence of active free radical generation as the radicals combine with NO to form peroxynitrite. In addition, generation of contractile agents via the increased free radical is a possibility. Other vascular dilatory mechanisms are not so easily compromised at least within a short duration of assault. In the present study, we have investigated the hypothesis that diabetes attenuates both NO-mediated endothelium-dependent and independent dilation. We now report that chronic hyperglycemia attenuates $\mathrm{NO}$ as well as isoproterenol, and BK induced dilations. This study shows that following 12 weeks of diabetes which results in chronic high glucose, alteration of vascular function goes beyond the traditional endothelium-dependent NO-mediated dilation. The reason behind this observation is still not known. But following chronic hyperglycemia, alteration of different vital proteins and receptors is possible and wide spread. Chronic exposure of the vasculature to hyperglycemia might have resulted in compensatory alteration of the vascular walls. Such alterations can manifest via altered protein synthesis and vascular enzymatic synthesis; such can affect the mode of vascular reactivity to vasoactive agents leading to wide spread alterations of numerous vascular dilatory mechanisms beyond the traditional endotheliumdependent vasodilators. This speculation is consistent with a few reported decreased in response to nitrovasodilators observed in diabetic rat which preceded a disturbed response to ACh (Dai et al, 1993). However, impaired endothelium-independent reactivity in diabetes was more frequently observed in humans (Williams et al, 1996, Clarkson et al, 1996, Lekakis et al, 1997) than in animals (Dai et al, 2000) than in animal models of diabetes. The reason for the differential extent of vascular dysfunction beyond $\mathrm{NO}$ / endothelium-dependent impairments could be due to the extent of hyperglycemia dysregulation observed in the animals before the experiments as compared to more advanced state of diabetes in man before studies reported. In our present studies reported, the experiments were performed 12 weeks following induction of diabetes as against $4-5$ weeks reported in most animal studies. The duration of the diabetic conditions in our studies could be comparable to the reported advanced diabetic conditions observed in human before the reported studies (Williams et al, 1996, Clarkson et al, 1996, Lekakis et al, 1997). Thus, our present results are consistent with that reported in humans and that such duration of treatment is necessary to provide comparable disease conditions in humans.

Under conditions of enhanced vascular stress such as in hypertension, diabetes, etc, compromised of NO-mediated vascular relaxation has been frequently reported especially following acute perturbation (Tesfamariam et al, 1991, Tesfamariam and Cohen, 1992; Yakubu et al, 2004; Sobrevia and Mann, 1997; Gutterman, 2002; Cosentino et al, 1997; Brodsky et al, 2001). Under acute perturbation changes to none-NO dependent vasodilatory mechanisms is not so common. For example, in acute glucose, attenuation of NO-mediated ACh relaxation has been reported while relaxation mediated by cAMP, prostanoid, EDHF, isoproterenol, papaverine, hydralazine etc have not been observed under this condition (Yakubu et al, 2004; Lucarelli and D'Onofrio, 1997). Under a condition of chronic oxidative stress, the elevated superoxide produced will continue to deprive the endothelium adequate supply of NO by converting it to peroxynitrite (Moncada et al, 1991; Rubanyi and Vanhoutte, 1986). Peroxynitrite so produced has contractile property as well as the ability to stimulate synthesis and release of vasoconstrictor agents such as angiotensin II and endothelin-1. These agents are mitogenic resulting in vascular remodeling, enhancing vascular responses to constrictor agents. In addition to the vascular remodeling, receptor sensitivity, numbers and or responsiveness of the receptor agent complex to efficient activation of the second messenger system may be compromised in the presence of heightened oxidative state. Consistent with such condition, vascular responses to the contractile agent (PE) were significantly enhanced in the STZ rats. All these together possibly could have contributed to the altered vascular responses to the non-endothelium dependent relaxations observed. Thus, STZ-induced impaired vasodilation may be mediated via chronic hyperglycemia-induced alteration of the vascular smooth muscle cell receptor proteins that may involve that recognized by BK, isoproterenol as well as their second messenger systems in addition to impairment of NO-dependent dilatory system. These findings suggest that distinct mechanisms 
may mediate the impaired response to endothelium-independent agonists and such impairment could be due to the extent and duration of blood glucose dysregulation. Thus, our present results are consistent with that reported in humans and that such duration (12 weeks) of treatment is necessary in rat to provide comparable disease conditions to that observed in humans.

\section{Reference}

1. Brodsky SV, Morrishow AM, Dharia N, Gross SS, Goligorsky MS. Glucose scavenging of nitric oxide. Am J Physiol (Renal Physiol) 2001; 280: F480-F486.

2. Chan NN, Vallan P, Colhoun HM. Nitric Oxide and vascular responses in type 1 diabetes. Diabetologia 2000; 43: 137-147.

3. Clarkson P, Celermajer DS, Donald AE, Sampson M, Sorensen KE, Adams M, Yue DK, Betteridge DJ, Deanfield JE. Impaired vascular reactivity in insulin-dependent diabetes mellitus is related to disease duration and low density lipoprotein cholesterol levels. $J$ Am Coll Cardiol 1996; 28: 573-579.

4. Cines DB, Pollak E, Buck CA, Loscalzo J, Zimmerman GA, McEver RP, Pober JS, Wick TM, Konkle BA, Schwartz BS, Barnathan ES, McCrae KR, Hug BA, Schmidt AM, Stern DM. Endothelial cell physiology and the pathophysiology of vascular disorders. Blood 1998; 91: 3527-3561.

5. Cohen RA, Vanhoutte PM. Endothelium-dependent hyperpolarization beyond Nitric Oxide and cyclic GMP. Circulation 1995; 92: 3337-3349.

6. Cosentino F, Hishikawa K, Katusic ZS, Lusher TF. High glucose increases nitric oxide synthase expression and superoxide anion generation in human aortic endothelial cells. Circulation 1997; 96: 25-28.

7. Dai F, Diederich A, Skopec J, Diederich D. Diabetes-induced endothelial dysfunction in streptozotocin-treated rats: role of prostaglandin endoperoxides and free radicals. J Am Soc Nephrol 1993; 4: 1327-1336.

8. De Vriese AS, Verbeuren TJ, Van de Voorde J, Lameire NH, Vanhoutte PM. Endothelial dysfunction in diabetes. Br J Pharmacol 2000; 130 (5): 963-974.

9. Ganz MB, Seftel A. Glucose-induced changes in protein kinase $\mathrm{C}$ and nitric oxide are prevented by vitamin E. Am J Physiol Endocrinol Metab 2000; 278: E146-E152.

10. Giugliano D, Ceriello A, Paolisso G. Oxidative stress and diabetic vascular complications. Diabetes Care 1996; 19: 257-267.

11. Giugliano D, Marfella R, Coppola L, Verazzo G, Acampora R, Giunta R, Nappo F, Lucarelli C, D'Onofrio F. Vascular effects of acute hyperglycemia in humans are reversed by L-arginine. Evidence for reduced availability of nitric oxide during hyperglycemia. Circulation 1997; 95; 1783-1790.
12. Gutterman DD. Vascular dysfunction in hyperglycemia. Is protein kinase C the culprit? Circ Res 2002; 90: 5-7.

13. Hoepfl B, Rodenwaldt B, Pohl U, de Wi C. EDHF, but not NO or prostaglandins, is critical to evoke a conducted dilation upon ACh in hamster arterioles. Am J Physiol 2002; 283 (3): H996-H1004.

14. Ignarro LJ. Biosynthesis and metabolism of endothelium-derived nitric oxide. Annu Rev Pharmacol Toxicol 1990; 30: 535-560.

15. Inoguchi T, Li P, Umeda F, Yu HY, Kakimoto M, Imamura M, Aoki T, Etoh T, Hashimoto T, Naruse M, Sano H, Utsumi H, Nawata H. High glucose and free fatty acid stimulate reactive oxygen species production through protein kinase $\mathrm{C}$-dependent activation of NAPD(P)H oxidase in cultured vascular cells. Diabetes 2000; 49: 1939-1945.

16. Lekakis J, Papamichael C, Anastasiou H, Alevizaki M, Desses N, Souvatzoglou A, Stamatelopoulos S, Koutras DA. Endothelial dysfunction of conduit arteries in insulin-dependent diabetes mellitus without microalbuminuria. Cardiovasc Res 1997; 34: 164-168.

17. Moncada S, Palmer RMJ, Higgs EA. Nitric Oxide: Physiology, Pathophysiology, and Pharmacology. Pharmacol Rev 1991; 43: 109-142.

18. Rubanyi GM, Vanhoutte PM. Super oxide anions and hyperoxia inactivate endothelium-derived relaxing factor. Am J Physiol 1986; 250: H822-H827.

19. Sobrevia L, Mann GE. Dysfunction of the endothelial nitric oxide signaling pathway in diabetes and hyperglycemia. Exp Physiol 1997; 82: 423-542.

20. Taylor PD, Graves JE, Poston L.Selective impairment of acetylcholine-mediated endothelium-dependent relaxation in isolated resistant arteries of the streptozotocin-induced diabetic rats. Clin Sci 1995; 88: 519-524.

21. Tesfamariam B, Brown ML, Cohen RA. Elevated glucose impairs endothelium-dependent relaxation by activating protein kinase C. J Clin Invest 1991; 87: 1643-1648.

22. Tesfamariam B, Cohen RA. Free radicals mediate endothelial cell dysfunction caused by elevated glucose. Am J Physiol 1992; 263: H321-H326.

23. Williams SB, Cusco JA, Roddy M, Johnstone MT, Creager MA. Impaired nitric oxide-mediated vasodilation in patients with non-insulindependent diabetes mellitus. J Am Coll Cardiol 1996; 27: 567-574.

24. Yakubu MA, Sofola OA, Igbo I, Oyekan AO. Link between free radicals and protein kinase $\mathrm{C}$ in glucose-induced alteration of vascular dilation. Life Sci 2004; 75 (24): 2921-2932.

Received March 16, 2010. Accepted December 18, 2011. 